\title{
REMARKS CONCERNING THE PAPER OF W. L. AYRES* ON THE REGULAR POINTS OF A CONTINUUM $\dagger$
}

\author{
BY \\ KARL MENGER
}

The reading of Ayres' interesting paper suggested to me the following remarks:

1. The order of a subset of a set $S$ in a point $p \ddagger$ cannot surpass the order of $S$ in $p$. Hence if $S^{2}$ denotes the set of all points of $S$ of order 2 , then $S^{2}$ has in each point of $S$ the order 2, the order 1, or the order 0 , where the terms "order 0 " and "0-dimensional" are used synonymously. $S^{2(0)}, S^{2(1)}, S^{\text {II }}$ may denote the set of all points of $S$ in which $S^{2}$ has the order $0,1,2$, respectively. The points of order 2 of $S$ are also called the ordinary points of $S$, and the set $S^{2}$ of all ordinary points of $S$ may be called the ordinary part of $S$. The set $S^{\mathrm{II}}$ of all ordinary points of the ordinary part of $S$ may be designated the ordinary kernel of $S$. We have

$$
S^{2}=S^{2(0)}+S^{2(1)}+S^{\pi} .
$$

Ayres has proved $\S$ that an ordinary point of a continuum $C$ in which the set $C^{2}$ is at least one-dimensional (i.e., in our terminology a point of $C^{2(1)}$ $+C^{\mathrm{II}}$ ), and which is besides a local cut point of $C$, is always an end point of a free arc of $C$; that is, of an arc which, after omission of its two end points, is open in $C$. Exactly in the same way it can be proved that a point $p$ of $C^{\text {II }}$, which is a local cut point of $C$, lies in the interior of a free arc of $C$. Ayres further proves\| that each point of $C^{2(1)}+C^{\text {II }}$, and therefore especially each point of the set $C^{\mathrm{II}}$, is a local cut point. It follows then that each point of the ordinary kernel of a continuum lies in the interior of a free arc.

2. If $C$ is a given continuum, let us call an ordinary element of $C$ a set $E$ which is open in $C$, homeomorphic with either the straight line or the circle, and which is saturated with respect to these properties; that is to say, $E$ is not a proper subset of a set $E^{\prime}$ which is open in $C$ and homeomorphic with a straight line or a circle. It is very easy to prove that two ordinary elements of a continuum are either identical or mutually exclusive, and that the system of all ordinary elements of a continuum is at most denumerable.

* These Transactions, vol. 33, pp. 252-262.

$\dagger$ Presented to the Society, June 13, 1931; received by the editors April 13, 1931.

$\ddagger$ Cf. the references concerning the concept of curve and of order at the end of this paper.

$\S$ Loc. cit., p. 257.

|| Loc. cit., p. 259. 
According to our first remark, each point of $C^{\mathrm{II}}$ is contained in a free arc of $C$ and therefore in an ordinary element of $C$. Conversely, each point of an ordinary element of $C$ is evidently a point of $C^{\mathrm{II}}$. Hence $C^{\mathrm{II}}$ is the sum of all ordinary elements of $C$ and we have proved the following

ORDINARY KERNEL THEOREM. If $C$ is a continuum, then the ordinary kernel $C^{\mathrm{II}}$ of $C$ is either identical with $C$ (which is the case if and only if $C$ is homeomorphic with the circle), or $C^{\mathrm{II}}$ is the sum of an at most denumerable system of sets, each of which is homeomorphic with the straight line, and each two of which are mutually exclusive. Further, each of the sets is open in $C$ and saturated with respect to the properties of being open in $C$ and homeomorphic with the straight line.

3. Let $E$ be an ordinary element of the continuum $C$ which is not identical with $C$, and therefore not homeomorphic with the circle, but homeomorphic with the straight line. The closed cover $\bar{E}$ of $E$ may be homeomorphic with the circle. In this case $\bar{E}-E$ contains exactly one point which is necessarily of an order greater than 2. As an example, $C$ may be the lemniscate with the cut point $p$ of order 4 , and $E$ may be one of the two components of $C-p$. The set $\bar{E}$ may be an arc; for instance, $C$ is an arc, and $E$, the arc diminished by its end points. It is obvious that $\bar{E}-E$ cannot contain more than two end points of $\bar{E}$, though the set $\bar{E}-E$ may contain more than two points, which are, however, necessarily irregular points of $C$. For an example of this consider for $C$ the curve $y=\sin (1 / x), 0<x \leqq 1$, and $-1 \leqq y \leqq 1, x=0$, and for $E$ the set $y=\sin (1 / x), 0<x<1$.

If $p$ is a point of $C^{2(1)}$, that is, an end point of the ordinary part of $C$, then $p$ does not lie in an ordinary element of $C$ since ordinary elements contain only ordinary points of the ordinary part of $C$. But $p$ is an end point of a free arc of $C$ and therefore a point of the closed cover of at least one ordinary element of $C$. As $p$ is a point of $C^{2}$ and hence a regular point of $C$, it must be an end point of the closed cover of an ordinary element of $C$. Since the set of all ordinary elements is at most denumerable, and the closed cover of each of them contains at most two end points, we have proved the following: The set $C^{2(1)}$ of all end points of the ordinary part of a continuum is, at most. denumerable.

4. The set $C^{2(0)}$ being 0 -dimensional, we have now a complete knowledge of the structure of the ordinary part of a continuum. We state this in the following

ORDINARY PART THEOREM. The ordinary part of a continuum is the sum of the ordinary kernel, the, at most, denumerable set of all end points, and the, at most, 0-dimensional set of all points in which it is 0-dimensional. 
5. If $S$ is a set and $p, q, r$ are three distinct points of a connected subset $S^{\prime}$ of $S$, then it is said that $q$ separates $S^{\prime}$ in $S$ between the points $p$ and $r$ if $S-q$ is the sum of two separated subsets containing $p$ and $r$ respectively. The definition would be applicable also to the case where $S^{\prime}$ is not connected, but would not be useful in this case.

We call the point $q$ of $S$ a separating point of $S$ if the component of $S$ that contains $q$ contains two points $p$ and $r$ such that $q$ separates this component in $S$ between $p$ and $r$. Further, we call a point $q$ of $S$ a cut point of $S$ if the component of $S$ containing $q$ is not connected when $q$ is deleted from this set. Evidently, a separating point of $S$ is a cut point of $S$, whereas a cut point of $S$ need not be a separating point of $S$. If $S$ is a continuum then the cut points and the separating points are identical.

G. T. Whyburn* calls the point $q$ of $S$ a locally separating point of $S$ provided that there exists a neighborhood $U$ of $p$ such that $q$ is a separating point of $\bar{u} \cdot S$. A point $q$ is called a local cut point of $S$ provided that there exists a neighborhood $U$ of $q$ such that $q$ is a cut point of $\bar{u} \cdot S$. From the relations between separating points and cut points it is evident that each locally separating point is a local cut point, but not conversely; whereas, if $S$ is especially a locally connected continuum, the local separating points and the local cut points are identical. $\dagger$

An important result of G. T. Whyburn's mentioned paper is that for each continuum, the set of all locally separating points of an order greater than 2 is, at most, denumerable. This theorem may be expressed as follows: $A l l$ locally separating points, with the exception of an, at most, denumerable set, are ordinary points. Ayres has proved that all points of $S^{2(1)}+S^{\mathrm{II}}$ are local cut points. As these points lie in free arcs of the continuum, they are even locally separating. The set of all ordinary points of $C$ which are not locally separating is therefore a subset of $C^{2(0)}$ and hence, at most, 0-dimensional. We may, therefore, say All points of a continuum, except those of an, at most, 0-dimensional set, are locally separating points. We formulate these two results (the first result somewhat weakened) in the following way:

For each continuum, the set of all ordinary points and the set of all locally separating points may be obtained from each other by omission of an, at most, 0-dimensional set.

6. Ayres has proved $\ddagger$ the following lemma: If $C$ is a continuum containing two end points, $a$ and $b$, such that each set that separates $a$ and $b$ in $C$ contains at least one ordinary point of $C$, then $C$ is an arc between $a$ and $b$.

* Monatshefte für Mathematik und Physik, vol. 36, pp. 305-314.

$\dagger$ Whyburn, loc. cit.

$\ddagger$ Loc. cit., p. 257. 
Evidently this condition is satisfied by each arc and therefore characteristic for the arcs among the continua. But it is of interest that it is also characteristic for the arcs among general (not closed) connected sets. We show that in order that a connected set be an arc between the two end points $a$ and $b$, it is necessary and sufficient that each set that separates $a$ and $b$ in $C$ contain at least one ordinary point.

Let $C$ be a connected set satisfying the condition. Then each point of $C$ that separates $a$ and $b$ is an ordinary point. Let $S(a, b)$ be the set of all points of $C$ separating $a$ and $b$ in $C$. According to Whyburn the set $a+b+S(a, b)$ may be ordered in the following way: $p$ is before $q$ if there exists a decomposition of $S-q$ into two separated subsets, one of which contains $a$ and $p$, while the other contains $b$. We prove now that the set $a+b+S(a, b)$ is closed (not merely closed in $C)$. If $p$ is a point of accumulation of $S(a, b)$ different from $a$ and $b$, then there exists a sequence of points $\left\{p_{n}\right\}(n=1,2, \cdots)$ of $S(a, b)$ converging towards $p$ and monotonic (in the sense of the order of $S(a, b)$ ). Let us assume the sequence to be monotonic increasing; that is, such that for each integer $n$ the point $p_{n}$ lies before $p_{n+1}$ and before $p$. If $C-p_{n}=A_{n}$ $+B_{n}$ is a decomposition of $C-p_{n}$ into two separated sets $\left(A_{n}\right.$ containing $a$, $B_{n}$ containing $\left.b\right)$, then the set $A=\sum_{n=1}^{\infty} A_{n}$ is open in $C$ and contains $a$, but not $b$, whereas $p$ lies in $\bar{A}-A$. Furthermore, it is easily proved that if the set $\bar{A}-A$ should contain any point besides $p$, then all points of $\bar{A}-A$ would be irregular points. Hence the point $b$, supposed to be an end point of $C$, would not lie in $\bar{A}-A$. Then the set $\bar{A}-A$ would separate $a$ and $b$ in $C$, and must contain, according to the hypothesis on $C$, at least one ordinary point, whereas all its points (provided that it contains a point different from $p$ ) are irregular. Hence $\bar{A}-A$ contains only the point $p$. As $C$ is connected, this point belongs to $C$. It separates $a$ and $b$ in $C$ and is therefore a point of $S(a, b)$ and an ordinary point of $C$. Since the set $S(a, b)$ is closed, it is identical with $C$. The set $C$ is therefore a continuum which, besides two end points, contains only points that separate these two end points, i.e., an arc. This completes the proof of the characterization of the arcs.

This characterization contains as a special case the characterization of the arcs among the general connected sets by the fact that besides two end points they contain only ordinary points.*

7. It seems probable to the writer that the methods of Ayres, as well as the preceding remarks, admit generalizations to higher genus. If $n$ is an integer and $\alpha$ an isolated ordinal number of the first or second class, then we say that the point $p$ of the compact space $C$ is of genus $\alpha$ and of order $n$ if $p$ is

* Cf. Frankl, Fundamenta Mathematicae, vol. 11 (1928), pp. 96-104. 
contained in neighborhoods, arbitrarily small, such that the $(\alpha-1)$ st derivatives of their boundaries contain at most $n$ points, whereas $p$ is not contained in arbitrarily small neighborhoods such that the $(\alpha-1)$ st derivatives of their boundaries contain less than $n$ points. ${ }^{*}$ It seems very likely that for each isolated ordinal number $\alpha$ the set of all points of a compact space of genus $\alpha$ and order $n$ is 0 -dimensional for each $n \neq 2$. It appears not impossible that even the set of all points of order $n$ for some isolated genus is 0 -dimensional if $n \neq 2$. As to the ordinary parts of higher genus, they admit decompositions analogous to the case of genus one treated in these remarks. As ordinary kernel of genus 2 of the continuum $C$ we also might define the ordinary kernel of the closed set $C-C^{\text {II }}$ and so on, by transfinite induction.

8. Finally, by way of more complete citation of the literature on the concepts and results of the theory of curves than is given in Ayres' paper, I quote in what follows the references concerning the concept of curve and of order of points given by the Encyklopädie der Mathematischen Wissenschaften, IIIAB 13, 1930 (Tietze-Vietoris), p. 234, footnote 276:

"K. Menger, Hinterlegung Nr. 778 (1921) bei der Wien. Akad. d. Wissensch.; Monatshefte f. Math. u. Phys. 33 (1923); Mathem. Annalen 95 (1925); Proc. Ac. Amsterdam 28 (1925); P. Urysohn, Paris C. R. 175 (1922); Fund. Math. 7 (1925)."

The characterization of the arc among the continua by the property that it contains besides two end points merely points of order 2 is mentioned without proof by Menger, Hinterlegung Nr. 778 (1921) (cf. Proceedings of the Amsterdam Academy, vol. 29, p. 1122, and Monatshefte für Mathematik und Physik, vol. 38, and by Urysohn, Paris Comptes Rendus, vol. 175 (1922). This theorem is proved by Menger, Mathematische Annalen, vol. 95 (1925), and by Urysohn, Amsterdam Verhandelingen, vol. 13 (1927).

* Cf. Menger, Fundamenta Mathematicae, vol. 10, pp. 96-115, and Reschovsky, Fundamenta Mathematicae, vol. 14.

Rice INSTITUTE, Houston, TEXAS 